\title{
A Spintronic Memristor Based PID Controller
}

\author{
Zhekang Dong ${ }^{1}$, Shukai Duan ${ }^{1}$, Xiaofang $\mathrm{Hu}^{2}$, Lidan Wang ${ }^{1}$, Fanyi Meng ${ }^{1}$ \\ ${ }^{1}$ School of Electronics and Information Engineering, Southwest University, Chongqing, 400715, China \\ ${ }^{2}$ Department of Mechanical and Biomedical Engineering, City University of Hong Kong, Kowloon, Hong Kong
}

\begin{abstract}
The spintronic memristor was proposed based upon the magnetic theory shortly after the discovery of the Hewlett-Packard (HP) TiO2 memristor. Different from that of the TiO2 memristor, the resistance of a spintronic memristor not only depends on the historic profile of voltage and current through the device, but also is closely related to the current density. In this work, we review the mathematical model of the spintronic memristive device based on magnetic theory and analyze its memristive effects in detail. Then we provide a corresponding Simulink model. Furthermore, by incorporating the spintronic memristor into a conventional PID control circuit, the paper investigates an implementation approach for a novel compact memristive PID controller. The findings of the study may contribute to further development of the modern control technology, especially in hardware realization of micro-control system. Finally, reported simulations demonstrate the effectiveness of the proposed scheme.
\end{abstract}

Keywords- spintronic memristor; PID control circuit; micro-control system.

\section{INTRODUCTION}

In 1971, Professor Chua defined the relationship between flux linkage and charge through a device based on the symmetry arguments of circuit theory and thus predicted the existence of the fourth fundamental circuit element, called memristor [1]. In 2008, the first realization of the memristor was illustrated in HP Laboratory, which garnered increasingly extensive interests from numerous researchers [2]. Soon later, three possible structures of spintronic memristors were proposed by Chen et al. [3, 4] in which the memristive effect was obtained by the spin-torque-induced magnetization switching or the magnetic-domain-wall motion. Unlike the solid-state $\mathrm{TiO}_{2}$ thin film device presented in [2], the performance of the spintronic memristor is more flexibly. Meanwhile, the magnetic device can be integrated into CMOS devices with exquisite technology and low cost [4]. As a promising nano-electronic, the spintronic device captures a number of unique properties that have found attractive potentials in various fields, such as nonvolatile memory, nonlinear circuit and system, neuromorphic system, signal processing, control, and learning system etc [5-7].

In the past several decades, the well-known proportional-integral-derivative (short for PID) controllers have been playing a significant role in the modern industry control area [8]. There is a growing popularity of PID controllers mainly on account of their simplicity and superior dynamic performance. Generally, the PID controllers, almost the linear fixed gain PID controllers, are adequate for controlling a physical process. The demands for high-performance control with environmental parameters or operating conditions varying are far beyond the capabilities of common PID controllers $[9,10]$. In this regards, many effective and feasible approaches have been proposed, which contain the self-tuning method, fuzzy logic, general predictive control and neural networks strategy, and so forth $[11,12]$. In this paper, we introduce the physical model of spintronic memristor, describe its mathematical model and Simulink model accordingly, and analyze the memristive characteristics according to numerical simulation. Moreover, a spintronic memristor takes the place of the linear resistor in the PID controller circuit, in so doing, the memristance could be adjusted flexibly with the transient current density varying, as a result, the spintronic memristive device realizes the tunable gain.

The rest of the paper is organized as below. In Section II, the mathematical model and Simulink model of the spintronic memristor based on magnetic-domain-wall motion mechanism are presented. The nonlinear adaptive PID controller based on spintronic memristor (abbreviated to SM-PID controller) is proposed in Section III. Next, the Section IV exhibits the simulation results and corresponding analysis of SM-PID controller. At last, the conclusions are given in Section V.

\section{THE SPINTRONIC MEMRISTOR MODEL}

\section{A. The mathematical model of spintronic memristor}

Among various existing spintronic memristor devices proposed in [4], the spintronic memristive device based upon magnetic-domain-wall motion is a promising option for its simple structure. Fig. 1 shows the physical structure of the spintronic memristor and its corresponding simplified equivalent circuit.

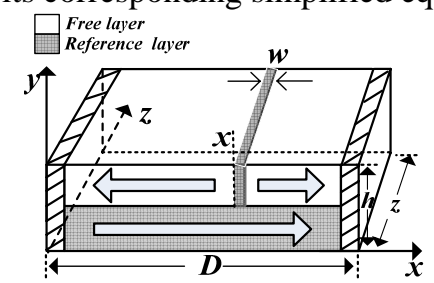

(a)

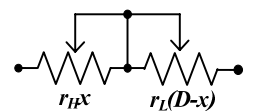

(b)
Figure 1. A spintronic memristor based on magnetic-domain-wall motion. (a) 3 dimension structure. (b) equivalent simplified circuit.

As shown in Fig. 1(b), the total resistance of the spintronic memristor, $M(x)$, is expressed as two resistors connected in series:

$$
M(x)=r_{H} \cdot x+r_{L} \cdot(D-x)
$$


where $r_{H}$ and $r_{L}$ represent the value of resistance per unit length when the segment of spin-valve strip is at the high- or low-resistance states, respectively. $D$ is the length of the device, $x$ represents the position of the domain-wall which can be calculated by the integral of the domain wall movement velocity $v$ over time $t$ as:

$$
x(t)=\int_{0}^{t} v d t=\int_{0}^{t} J \cdot \Gamma_{v} d t
$$

where $\Gamma_{v}$ is the so-called domain wall velocity coefficient, its value depends on the device structure and manufacture materials. The relationship between the domain wall movement velocity $v$ and current density $J$ can be expressed as:

$$
J(t)=\frac{V(t)}{M(t) \cdot h \cdot z}
$$

here $V(t)$ denotes the input voltage, $z$ and $h$ denote the width and the thickness of spin-valve strip, respectively.

Combining (1), (2) and (3), the resistance of spintronic memristor can be rewritten as:

$$
M(q)=r_{L} \cdot D+\left(r_{H}-r_{L}\right) \Gamma q(t)
$$

where $\Gamma=\frac{\Gamma_{v}}{h \cdot z}$ denotes the ratio between domain wall velocity coefficient and cross section of the spin-valve strip.

It is noteworthy that the domain wall movement in the spintronic memristor will not occur until the current density $J(t)$ is larger than the critical current density $J_{c r}$ [3]. Integrating (2), we can get:

$$
v= \begin{cases}J \cdot \Gamma_{v} & J \geq J_{c r} \\ 0 & J<J_{c r}\end{cases}
$$

\section{B. The Simulink model of spintronic memristor}

For the sake of analyzing the characteristics of spintronic memristor comprehensively, its Simulink model is built upon (1)-(5) and demonstrated in Fig.2. The model mainly composed of input and output modules, internal operation modules (multipliers and adders) and condition judgment modules. The parameters of the memristive device are set as $r_{H}=6 K M \Omega \quad, \quad r_{L}=4 K M \Omega \quad$, $x_{0}=0.3 \times 10^{-6} \mathrm{~m}, \Gamma_{v}=1.3517 \times 10^{-11}, J_{c r}=5 \times 10^{7} \mathrm{~A} / \mathrm{cm}^{2}$, $D=1000 \mathrm{~nm}, h=7 \mathrm{~nm}, z=10 \mathrm{~nm}$. The input signal applied into spintronic memristor is a sinusoidal voltage with an amplitude of $1.05 \mathrm{~V}$ and a frequency of $10 \mathrm{MHz}$.

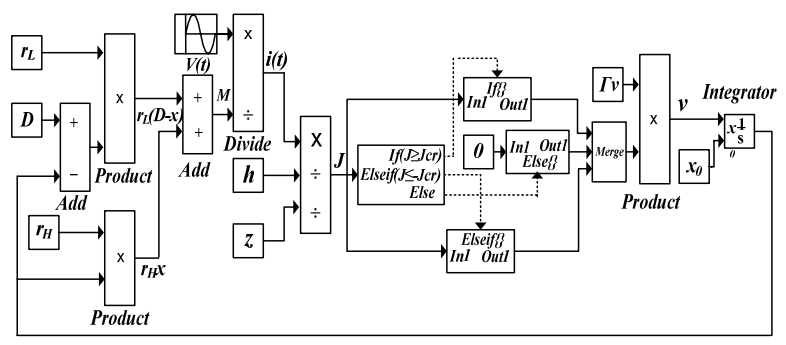

Figure 2. The Simulink model of the spintronic memristor
The Simulink results are shown in Fig 3. Fig. 3(a) exhibits the voltage of the memristor. The classic hysteresis loop in Fig. 5(b) shows that the spintronic memristor possesses the typical memristive effect.

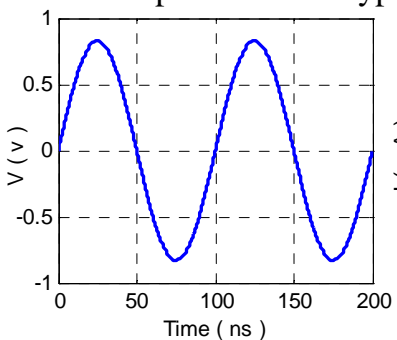

(a)

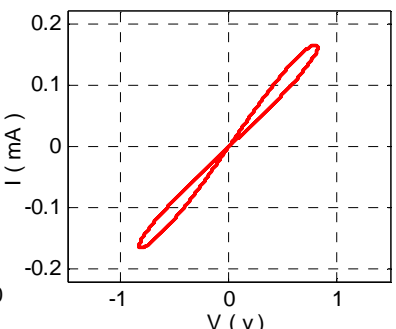

(b)
Figure 3. The simulation results of the spintronic memristor Simulink model

\section{CIRCUIT MODEL OF THE NONLINEAR PID CONTROLLER BASED ON SPINTRONIC MEMRISTOR}

Fig. 4 shows a normal linear PID controller circuit, where $u_{1}$ is the input and $u_{2}$ is the corresponding output which can be regarded as the control action $u(t) . C_{1}, C_{2}$, $R_{1}$, and $R_{2}$ are the constant capacitors and resistors in the circuit, respectively. The rest part, $O P A$, is an amplifier.

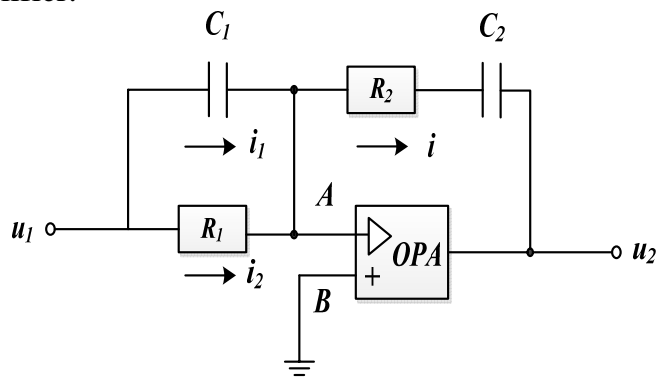

Figure 4. A schematic of the conventional PID controller circuit

Normally, the resistance of $O P A$ is so large that the current passing through $O P A$ can be assumed very weak, hence, we can consider the potential of point $A$ is equivalent to that of point $B$. Finally, we obtain the relationship between the input $u_{1}$ and the output $u_{2}$ as below:

$$
\begin{aligned}
u_{2}(t) & =R_{2} i(t)+u C_{2}(t) \\
& =\left(\frac{R_{2}}{R_{1}}+\frac{C_{1}}{C_{2}}\right) u_{1}(t)+\frac{1}{R_{1} C_{2}} \int_{0}^{t} u_{1}(\tau) d \tau+R_{2} C_{1} \frac{d u_{1}(t)}{d t}
\end{aligned}
$$

here $i(t)$ is the current flowing past resistor $R_{2}$.

There are a total of three terms: proportional, integral and derivative item respectively in (6). Thus the circuit exhibited in Fig. 4 could be utilized as a PID controller. Additionally, the values of the $C_{1}, C_{2}, R_{1}$, and $R_{2}$ are fixed electronic components, therefore, the proposed PID controller displayed in Fig. 4 of course is a kind of linear fixed gain controller.

The main idea of the SM-PID controller is to replace the resistor $R_{1}$ with a spintronic device whose resistance can change adaptively with the current density changing, as described in Fig.5. It is worth noting that the current density is the ratio of the current $i_{2}(t)$ and the cross-sectional area of the spintronic memristor in the circuit. 


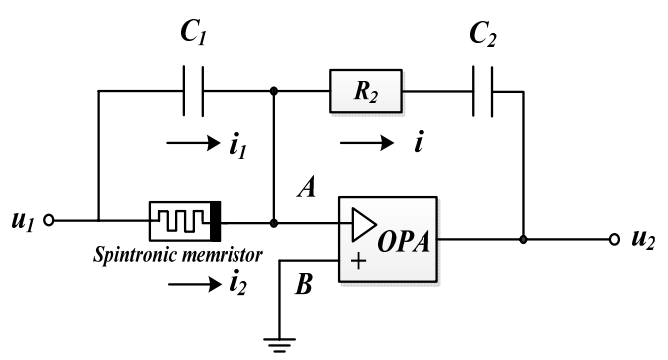

Figure 5. The SM-PID controller circuit

The current passing through the spintronic memristive device is:

$$
i_{2}(t)=\frac{u_{1}(t)}{M(q)}=\frac{u_{1}(t)}{r_{H} \cdot D+\left(r_{H}-r_{L}\right) \Gamma q(t)}
$$

The current flowing through $R_{2}$ and $C_{2}$ is:

$$
\begin{aligned}
i(t) & =i_{1}(t)+i_{2}(t) \\
& =C_{1} \frac{d u_{1}(t)}{d t}+\frac{u_{1}(t)}{r_{H} \cdot D+\left(r_{H}-r_{L}\right) \Gamma q(t)}
\end{aligned}
$$

Finally, the output $u_{2}$ can be calculated as: $u_{2}(t)=R_{2} i(t)+u C_{2}(t)$

$$
\begin{aligned}
= & R_{2} C_{1} \frac{d u_{1}(t)}{d t}+\frac{R_{2} u_{1}(t)}{r_{H} \cdot D+\left(r_{H}-r_{L}\right) \Gamma q(t)}+\frac{C_{1}}{C_{2}} u_{1}(t) \\
& +\frac{1}{C_{2}} \int_{0}^{t} \frac{u_{1}(\tau)}{r_{H} \cdot D+\left(r_{H}-r_{L}\right) \Gamma q(\tau)} d \tau
\end{aligned}
$$

Equation (9), the mathematical model of the nonlinear SM-PID controller, also consisted of the three parts: proportional item, integral item, and derivative item, respectively. The participation of the spintronic memristor lead to the nonlinear PID controller. As the current density varies, the resistance of the memristor changes correspondingly, which offers the tunable gain.

\section{NUMERICAL SIMULATIONS}

In this section, two examples are considered to validate the feasibility of the SM-PID controller.

\section{A. Error detection}

In practice, various error signals in control systems almost are impulsive signals, hence we firstly investigate its dynamics response of the SM-PID controller in the impulsive signal environment.

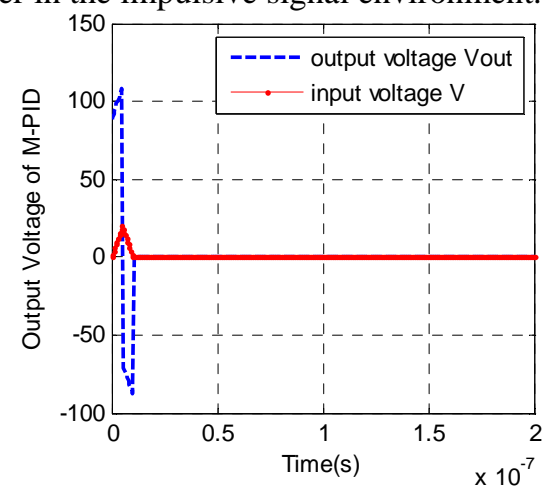

Figure 6. The relationship curve between input and output voltage when $u_{1}(t)=\delta t$
In Fig. 6, compared with the conventional linear PID controller, the output of the SM-PID controller reacts more rapidly (response time $t<0.3 \times 10^{-7} s$ ) and more shapely, which assists supervisors in finding and handling out the noise signals in time, and making sure the security of the entire control system.

\section{B. Control of a second-order system}

Making use of the proposed Simulink model of the spintronic memristor, we design a Simulink model of the SM-PID controller, as shown in Fig. 7(a), in which the SM module is the package of the spintronic memristor. Taking a example for a certain industrial application, we assume its mathematical model is a second-order system:

$$
f(s)=\frac{10}{2 s^{2}+4 s+5}
$$

The main parameters are set as: $R_{2}=2 K \Omega$, $C_{1}=C_{2}=0.5 \mathrm{mF}$. The input signal is chosen as a square wave with an amplitude of $1 \mathrm{~V}$, and its pulse width is $100 \mathrm{~ns}$.

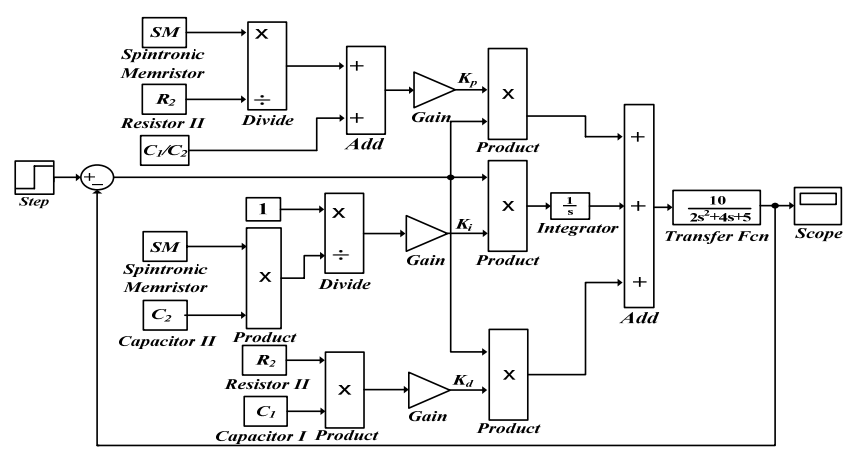

(a)

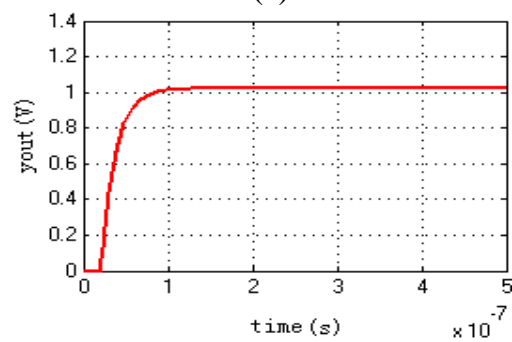

(b)

Figure 7. The control of the second-order system. (a) The Simulink model of the SM-PID controller. (b)The simulation result of the SM-PID controller

The simulation result is shown in Fig. 7(b). From the result, we can observe the plant has been successfully controlled by the SM-PID, in other words, the controlled system can achieve stable state quickly. The effectiveness is thus verified.

\section{V.CONCLUSIONS}

The paper describes the mathematical model of the spintronic memristor, presents its Simulink model in detail. The SM-PID controller that we combine the emerging spintronic memristor with the convention PID controller circuit by replacing one resistor in the circuit with a spintronic device and thus construct the novel 
model of the SM-PID controller. Compared with the conventional PID controllers, the proposed novel nonlinear PID controller owns more advantageous characteristics: (1) Its nanoscale physical implementation could promote the development of the micro-controller. (2) The response speed is large, which makes the response time usually last an order of magnitude of $10^{-7}$. (3) Because of the participation of the spintronic memristor, the proposed SM-PID controller realizes the self-adjustment of parameters, and so forth. Relevant simulation results verify the effectiveness of the combination scheme of the spintronic memristor and conventional PID controller.

\section{ACKNOWLEDGEMENTS}

The research work was supported by Program for New Century Excellent Talents in University, National Natural Science Foundation of China (Grant Nos. 61372139, 61101233, 60972155, 61374078), “Spring Sunshine Plan" Research Project of Ministry of Education of China (Grant No. z2011148), Technology Foundation for Selected Overseas Chinese Scholars, Ministry of Personnel in China (Grant No. 2012-186).

\section{REFERENCES}

[1] L. O. Chua. Memristor - the missing circuit element. IEEE Transactions. Circuit Theory, 1971, 18(5): 507-519.

[2] D. B. Strukov, G. S. Snider, D. R. Stewart, R. S. Williams. The missing memristor found. Nature, 2008, 453 (7191):80-83.
[3] Y. R. Chen, X. B. Wang, Compact modeling and corner Analysis of spintronic memristor. IEEE/ACM International Symposium on Nanoscale Architectures, 2009:7-12.

[4] X. B. Wang, Y. R. Chen, H. W. Xi, D. Dimitrov. Spintronic memristor through spin-torque-induced magnetization motion. IEEE Electron Device Letters, 2009, 30(3):294-297.

[5] X.F. Hu, S.K. Duan, L.D. Wang. Memristive multilevel memory with applications in audio signal storage. Lecture Notes in Computer Science. 2011, 7002(1):228-235.

[6] L.D. Wang, M.T. Duan, S.K. Duan. Memristive chebyshev neural network and its applications in function approximation. Mathematical Problems in Engineering. Vol.2013.

[7] S.K. Duan, X.F. Hu, L.D. Wang, C.D. Li, P. Mazumder. Memristor based RRAM with applications. Science China Information Sciences.Vol.55,No.6, pp:1446-1460,2012.

[8] H.P. Jin, W.S. Su, Lee I-B. An enhanced PID control strategy for unstable processes. Automatic, 34(1998), 751-756.

[9] H. W. Wang, H. X. Liu , J.B. Yang, Dynamic analysis of a two-stage supply chain-a switched system theory approach, The International Journal of Advanced Manufacturing Technology. 43(2009) 200-210.

[10] Z. Zeng, J. Wang, Analysis and design of high-capacity associative memories based on class of discrete-time recurren neural networks, IEEE Transactions on System Manufacturing Cybernetics. 38(2008) 1525-1536.

[11] L.D. Wang, X.Y. Fang, S.K. Duan, X.F. Liao. PID controller based on memristive CMAC network. Abstract and Applied Analysis. Vol.2013, ID.510238, 6 pages, 2013.

[12] X.Y. Fang, L.D. Wang, S.K. Duan. Adaptive single-neuron proportional integral derivative controller base on memristor. Applied Mechanics and Materials. Vol.284-287, pp: 2205-2209, Jan 2013. 